\title{
From Hair to Heart: Hair-Follicle-Associated Pluripotent (HAP) Stem Cells Can Simultaneously Differentiate Into Mature Cardiomyocytes and Atrial Myocytes
}

Michiko Yamane ( $\sim$ mm20054@st.kitasato-u.ac.jp )

Kitasato University School of Medicine

Nanako Takaoka

Kitasato University School of Medicine

Koya Obara

Kitasato University School of Medicine

Kyoumi Shirai

Kitasato University School of Medicine

Ryoichi Aki

Kitasato University School of Medicine

\section{Yuko Hamada}

Kitasato University School of Medicine

Nobuko Arakawa

Kitasato University School of Medicine

Manabu Tanaka

Bio-Imaging Center, Kitasato University School of Medicine

Robert M. Hoffman

AntiCancer (United States)

Yasuyuki Amoh

Kitasato University School of Medicine

\section{Research Article}

Keywords: Hair follicle, HAP stem cells, stem cell, simultaneous differentiation, atrial myocytes, beating cardiomyocytes

Posted Date: November 3rd, 2021

DOI: https://doi.org/10.21203/rs.3.rs-1001809/v1 
License: (c) (i) This work is licensed under a Creative Commons Attribution 4.0 International License. Read Full License 


\section{Abstract}

Hair-follicle-associated pluripotent (HAP) stem cells express nestin, and are located in the bulge area of hair follicles and can differentiate to numerous types of cells. In the present study, we demonstrate that rat HAP stem cells simultaneously differentiated to mature cardiomyocytes and atrial myocytes. The addition of isoproterenol, activin A, bone morphogenetic protein 4 (BMP 4), basic fibroblast growth factor (bFGF), and cyclosporin A (CSA), induced simultaneously differentiation of HAP stem cells to c-kitpositive cardiomyocytes and MLC-2a-expressing atrial myocytes. The results of the present study suggest that HAP stem cells differentiating to cardiomyocytes and atrial myocytes have future clinical potential for heart regeneration.

\section{Introduction}

Cardiomyocytes are a major target in regenerative medicine. In recent years, there are intense efforts have been made to induce cardiomyocyte differentiation from embryonic stem (ES) cells and induced pluripotent stem cells (iPSC). However, there are major issues in terms of maturation and functionality of the differentiated cardiomyocytes derived from these two types of stem cells. [1, 2, 3]. Nestin-expressing hair-follicle-associated pluripotent (HAP) stem cells are found in the hair-follicle bulge area and can differentiate into neurons, glia, keratinocytes, smooth muscle cells, melanocytes, and beating cardiac muscle cells $[4,5,6,7,8]$. HAP stem cells from mice have been used to repair the severed sciatic nerve in mouse models $[9,10,11,12,13,14]$. The implanted HAP stem cells differentiated into Schwann cells in the re-joined nerve and restored nerve and leg function. HAP stem cells have been used to repair the severed spinal cord in mice leading to improved hind limb locomotion [10, 15]. Isoproterenol stimulated mouse HAP stem cells to differentiate to cardiac-muscle cells in large numbers in culture. The addition of activin A, BMP4, and bFGF, along with isoproterenol, stimulated the cardiac-muscle cells to form tissue sheets of beating heart muscle cells [5]. In the present study, we demonstrate that rat HAP stem cells can simultaneously differentiate into mature cardiomyocytes and atrial myocytes with regenerative potential.

\section{Materials And Methods}

\section{F344 Rat}

Four weeks old F344/jcl female rat (50 g or more) (CLEA Japan, Tokyo, Japan) were used to isolate vibrissa hair follicles. The experimental animals were kept in an animal housing system maintained at 24 $\pm 1^{\circ} \mathrm{C}$, relative humidity of $50-60 \%$, and 14 hours of light 10 hours of dark intervals. The experimental protocol was approved by the Kitasato University School of Medicine Animal Care Committee (No. 2020035). The study was conducted according to the ARRIVE guidelines for the reporting of animal experiments. All methods complied with the guidelines for the proper conduct of animal experiments of the Science Council of Japan.

Isolation and division of rat vibrissa hair follicles. 
Isolation and division of rat vibrissa hair follicles were performed as previously reported [22]: For isolation of vibrissa hair follicles from the F344/jcl rats, the animals were anesthetized with a combination of $0.375 \mathrm{mg} / \mathrm{kg}$ medetomidine, $2.0 \mathrm{mg} / \mathrm{kg}$ midazolam and $2.5 \mathrm{mg} / \mathrm{kg}$ butorphanol. The upper lip containing the vibrissa pad was cut, and inner surface was exposed. All vibrissa hair follicles were gently pulled out from the pad, one by one, with fine forceps, under a binocular microscope. The tissues around the isolated hair follicles were removed, and the hair follicles were divided into thirds. All surgical procedures were performed in a sterile environment.

\section{Induction of cardiomyocyte and atrial-myocyte differentiation}

The upper third parts of rat vibrissa hair follicles were placed on Matrigel ${ }^{\circledR}$ Matrix (\#356231, Corning Incorporated, Corning, NY, USA) culture in $27 \mathrm{~mm}$ glass-bottom culture dishes (\#BC-SFGD27, Bio Medical Science, Tokyo, Japan), in DMEM (\#D6429, Sigma-Aldrich, St. Louis, MO, USA) containing 10\% fetal bovine serum (FBS), 50 mg/ml gentamycin (\#15750-060, GIBCO, Grand Island, NY, USA), 2 mM Lglutamine (\#25030, GIBCO) ,10 mM HEPES (\#H0887, Sigma-Aldrich). To induce cardiomyocyte differentiation, isoproterenol (3 $\mu \mathrm{M})$ (\#16504, Sigma-Aldrich), activin A (10 ng/ml) (\#338-AC-010, HumanZyme, Chicago, IL, USA), BMP4 (10 ng/ml) (\#120-05ET, HumanZyme,) and bFGF (5 ng/ml) (\#GF003, Millipore, Temecula, CA, USA) were added to 10\% FBS-DMEM for 7 days. Afterward, to study the effect of CSA on cardiomyocytes differentiation, CSA $(1 \mu \mathrm{g} / \mathrm{mL}$ ) (\#AG-CN2-0079, AdipoGen Life Sciences, San Diego, CA, USA) was added to $10 \%$ FBS-DMEM for 14 days. The culture period was 21 days (Fig. 5). Medium was replaced every 3 days. Beating cardiomyocytes and atrial myocytes were recorded with a video camera (VCE-i700T, Shodensha, Osaka, Japan), attached to a microscope CKX53 (Olympus Corporation, Tokyo, Japan).

\section{Immunofluorescence staining}

Immunofluorescence staining was performed as previously reported [4]. Primary antibodies used were: anti-cardiac troponin I (cTnl) rabbit polyclonal antibody (1:200, \#PAA478Ra01, Cloud-Clone Corp, Houston, TX, USA); anti-MLC-2a mouse monoclonal antibody (1:40, \#565496, BD Biosciences, San Jose, CA, USA); anti-c-kit polyclonal antibody (1:200, \#bs-0672R, Bioss Antibodies, Woburn, MA, USA).

Secondary antibodies used were: goat anti-rabbit IgG conjugated with Alexa Flour ${ }^{\circledR} 568$ (1:400, \#A-21069, Invitrogen, Waltham, MA, USA); goat anti-mouse IgG conjugated with Alexa Fluor ${ }^{\circledR} 488$ (1:400, \#A-11001, Invitrogen). 4', 6-diamino-2-phenylindole, dihydrochloride (DAPI) (\#SE196, DOJINDO, Kumamoto, Japan) was used for counter staining. The images of stained cells were obtained with a LSM 710 microscope (Carl Zeiss, Oberkochen, Germany). The images were analyzed by LSM software ZEN (Carl Zeiss).

\section{Quantitative Polymerase Chain Reaction (qPCR)}

Total RNA was extracted from 21day cardiomyocytes using the RNeasy Plus Mini Kit (\#74134, Qiagen, Hilden, Germany) and then cDNA were synthesized with QuantiTect ${ }^{\circledR}$ Reverse Transcription (\#205311, Qiagen) according to the manufacturer's instructions. GAPDH was used to normalize gene expressions. Quantitative PCR was performed using the Power SYBRß Green PCR Master mix (\#4367659, Applied 
Biosystems, Waltham, MA, USA) on a CFX96 Real-Time PCR Detection System (Bio-Rad, Hercules, CA, USA) and analyzed by the Delta Delta Ct method. Forward and reverse primer sequences are shown in Table. 1.

\section{$\mathrm{Ca}^{2+}$ Imaging}

Intracellular calcium imaging was performed as previously reported [5]: The cells were washed with Earle's balanced salt solution (\#14155-063, GIBCO) with $2 \mathrm{mmol} \mathrm{CaCl}_{2}$ at $37^{\circ} \mathrm{C}$ and were loaded with the calcium-sensitive dye Fluo 4-AM (1 $\mu \mathrm{M})$ (\#F312, DOJINDO) and AM ester-dissolving reagent Pluronic F127 (0.04\%) (\#59004, FUJIFILM Wako, Osaka, Japan) for $20 \mathrm{~min}$ at $37^{\circ} \mathrm{C}$. Fluo 4-AM fluorescence (excitation at $495 \mathrm{~nm}$ and emission at $518 \mathrm{~nm}$ ) of beating cardiomyocytes was measured with a LSM 710 microscope (Carl Zeiss), were analyzed by LSM soft-ware ZEN Time series (Carl Zeiss).

\section{Transmission Electron Microscopy}

Differentiated cells from HAP stem cells were pre-fixed with $2.5 \%$ glutaraldehyde in $0.1 \mathrm{M}$ sodium cacodylate buffer for 30 min at $4^{\circ} \mathrm{C}$, post-fixed with $1 \%$ osmium tetroxide for 1 hour at $4{ }^{\circ} \mathrm{C}$. A biological specimen was immersed in a solution of $2 \%$ uranyl acetate for 15 min an aqueous solution after postfixation, for en-bloc staining. The specimens were then dehydrated through graded ethanol and embedded in epoxy resin. Ultrathin sections $(80 \mathrm{~nm})$, double-stained with $2 \%$ uranyl acetate and Reynold's lead citrate, were examined under electron microscopy H-7650 (Hitachi, Tokyo, Japan).

\section{Image processing}

Image processing was with image $\mathrm{J}$ software (National Institutes of Health, Bethesda, MD, USA) (Rasband, W.S. ImageJ, U. S. National Institutes of Health. Bethesda, MD, USA, 351 Available online: http://imagej.nih.gov/ij/, 1997-2012(accessed on 15 September 2021).). cTnl images were set to threshold from 50 to 255. MLC-2a images were set to threshold from 40 to 255.

\section{Statistical Analysis}

The experimental data are expressed as the mean SD. Statistical analyses were performed with the unpaired Student's t-test. A probability $(P)$ value of $p \leq 0.05$ was considered significant.

\section{Results}

\section{Rat HAP stem cells simultaneously differentiate into mature cardiomyocytes and atrial myocytes}

HAP stem cells were cultured in medium supplemented with isoproterenol, activin A, BMP4, bFGF, and CSA for 21 days. Differentiated beating cardiomyocytes (S1 Movie), expressing cardiac-troponin-I (cTnI), myosin-light-chain-2a (MLC-2a) and c-kit appeared in this medium (Fig. 1a, 1b). The percentage of HAP stem cells differentiating to cardiomyocytes in supplemented medium was $20.0 \pm 5.50 \%$ compared to $1.33 \pm 0.35 \%$ in non-supplemented medium $(p<0.005)(n=4)$. HAP stem cells simultaneously 
differentiated to atrial myocytes at $7.93 \pm 1.34 \%$ compared to $1.38 \pm 0.14 \%$ in non-supplemented medium $(p<0.05)(n=3)$ (Fig. 2a and 2b). The relative expression of mature cardiomyocyte-related sarcomere protein-encoding genes including cardiac-troponin-T (cTnT) and cTnl in differentiated from HAP stem cells cultured in supplemented medium compared to non-supplemented medium. cTnT; $6.02 \pm 0.57$, cTnl; $3.12 \pm 0.31(p<0.005)$. The relative expression of atrial myocyte related gene MLC-2a also significantly increased in supplemented medium. MLC-2a; $2.37 \pm 0.21(p<0.05)(n=3)$ (Fig. $2 c)$.

\section{Beating cardiomyocytes differentiated from HAP stem cells showed regular amplitude of intracellular calcium levels}

Intracellular $\mathrm{Ca}^{2+}$ concentration changes were associated with beating of the cardiomyocytes differentiated from rat HAP stem cells (Fig. 3). Phase-contrast microscopy of the cardiomyocytes loaded with the calcium-sensitive dye Fluo 4-AM showed time-dependent intensity changes (S2 Movie).

\section{Ultrastructural analysis of HAP stem-cell-derived mature cardiomyocytes}

We confirmed features of cardiomyocytes differentiated from HAP stem cells at the ultrastructural level using transmission electron microscopy. Rat HAP stem cells cultured in supplemented medium, derived cardiomyocytes resembled native cardiomyocytes, showing myofibrillar bundles with regularly aligned Zbands, A-and I-bands with a H-zone, enriched mitochondria, and T-tubule-like structures (Fig. 4).

\section{Discussion}

In the present report, we demonstrate that rat HAP stem cells cultured with isoproterenol, activin A, BMP 4, bFGF, and CSA can simultaneously differentiate into cardiomyocytes and atrial myocytes. Yan et al. previously demonstrated that addition of an immunosuppressant, cyclosporin-A (CSA), to mesoderm cells expanded cardiac progenitor cells and cardiomyocytes [16]. In the present report, cardiomyocytes differentiated from HAP stem cells were observed to contain T-tubules, sarcomere structures, and mitochondria, which are essential for mature cardiomyocytes [17, 18]. Furthermore, cardiomyocytes differentiated from rat HAP stem cells showed intracellular $\mathrm{Ca}^{2+}$ increasing with autonomous beating with a regular amplitude. Borysova et al. previously reported that right-atrial-appendage biopsy specimens from rats showed regular $\mathrm{Ca}^{2+}$ waves of approximately every 0.2 seconds [19], suggesting that cardiomyocytes differentiated from rat HAP stem cells were electrophysiological similar to rat adult atrial muscle. Cardiomyocytes differentiated from HAP stem cells were c-kit-positive. c-kit is a cardiac stem cell marker, and c-kit-positive cardiac stem cells are abundant in the atrial muscle in humans [20]. Based on this evidence, we consider that c-kit-positive atrial myocytes differentiated from HAP stem cells have characteristics of cardiac stem cells. HAP stem cells also differentiated to MLC-2a expressing atrial myocytes. In the present study, we succeeded in simultaneously inducing c-kit-positive cardiomyocytes and MLC-2a-expressing atrial myocytes from HAP stem cells. Atrial myocytes differentiated from HAP stem cells have characteristics of both cardiac stem cells and adult cardiomyocytes, and may be useful for regeneration of damaged myocardium. The possibility of clinical use of HAP stem cells for cardiac 
regeneration is feasible and practical, since HAP stem cells are readily available from everyone and can be cryopreserved and banked without loss of pluripotency $[8,21]$.

\section{Declarations}

\section{Acknowledgements.}

This work was partially supported by Grant-in-Aid for Scientific Research (C) $19 \mathrm{~K} 08781$ from the Ministry of Education, Science, Sports, and Culture of Japan, and the Terumo Life Science Foundation (to Amoh, Y.).

\section{Author contributions}

M.Y. experimental design, collection and assembly of data, data analysis and interpretation, manuscript writing. N.T., K.O., K.S. and R.A. data analysis and interpretation.

Y.H., N.A. and M.T. experimental design, collection and assembly of data. R.M.H. experimental design, manuscript writing, and final approval of manuscript. Y.A. experimental design, data analysis and interpretation, financial support, administrative support, manuscript writing, final approval of manuscript. All authors have read and agreed to the published version of the manuscript.

\section{Additional Information}

Publisher's note

Springer Nature remains neutral with regard to jurisdictional claims in published maps and institutional affiliations.

\section{References}

1. Snir, M. et al. Assessment of the ultrastructural and proliferative properties of human embryonic stem cell-derived cardiomyocytes. Am. J. Physiol. Heart Circ. Physiol. 285, H2355-2363 (2003).

2. Chanthra, N. et al. A Novel Fluorescent Reporter System Identifies Laminin-511/521 as Potent Regulators of Cardiomyocyte Maturation. Scientific Reports. 10, 4249 (2020).

3. Miki, K. et al. ERRy enhances cardiac maturation with T-tubule formation in human iPSC-derived cardiomyocytes. Nature Communications. 12, 3596 (2021).

4. Yashiro, M. et al. From hair to heart: Nestin-expressing hair-follicle-associated pluripotent (HAP) stem cells differentiate to beating cardiac muscle cells. Cell Cycle. 14, 2362-2366 (2015).

5. Yamazaki, A. et al. Isoproterenol directs hair follicle-associated pluripotent (HAP) stem cells to differentiate in vitro to cardiac muscle cells which can be induced to form beating heart-muscle tissue sheets. Cell Cycle. 15, 760-765 (2016).

6. Hoffman, R. M. The pluripotency of hair follicle stem cells. Cell Cycle. 5, 232-233 (2006). 
7. Amoh, Y. et. al. Human and mouse hair follicles contain both multipotent and monopotent stem cells. Cell Cycle. 8, 176-177 (2009).

8. Obara, K. et. al. Hair-follicle-associated pluripotent stem cells derived from cryopreserved intact human hair follicles sustain multilineage differentiation potential. scientific reports. 9, 9326 (2019).

9. Amoh, Y. et. al. Implanted hair follicle stem cells form Schwann cells which support repair of severed peripheral nerves. Proc. Natl. Acad. Sci. USA. 102, 17734-17738 (2005).

10. Amoh, Y., Li, L., Katsuoka, K. \& Hoffman, R. M. Multipotent hair follicle stem cells promote repair of spinal cord injury and recovery of walking function. Cell Cycle. 7, 1865-1869 (2008).

11. Amoh, Y., Hamada, Y., Aki, R., Kawahara, K., Hoffman, R. M. \& Katsuoka, K. Direct transplantation of uncultured hair-follicle pluripotent stem (hfPS) cells promotes the recovery of peripheral nerve injury. Cell Biochem. 110, 272-277 (2010).

12. Amoh, Y. et. al. Nestin-positive hair follicle pluripotent stem cells can promote regeneration of impinged peripheral nerve injury. J. Dermatol. 39, 33-38 (2012).

13. Amoh, Y., Katsuoka, K. \& Hoffman, R. M. Peripheral-nerve and spinal-cord regeneration in mice using hair-follicle-associated pluripotent (HAP) stem cells. Methods Mol. Biol 1453, 21-32 (2016).

14. Yamazaki, A. et. al. Implanted hair-follicle-associated pluripotent (HAP) stem cells encapsulated in polyvinylidene fluoride membrane cylinders promote effective recovery of peripheral nerve injury. Cell Cycle. 16, 1927-1932 (2017).

15. Liu, F. et. al. The bulge area is the major hair follicle source of nestin-expressing pluripotent stem cells which can repair the spinal cord compared to the dermal papilla. Cell Cycle. 10, 830-839 (2011).

16. Yan, P. et al. Cyclosporin-A potently induces highly cardiogenic progenitors from embryonic stem cells. Biochem Biophys Res Commun. 379, 115-120 (2009).

17. Guo, Y. \& Pu, W. Cardiomyocyte Maturation: New Phase in Development. Circ Res. 126, 1086$1106(2020)$.

18. Park, S. H., Kim, A., An, J., Cho, H. S. \& Kang, T. M. Nanoscale imaging of rat atrial myocytes by scanning ion conductance microscopy reveals heterogeneity of T-tubule openings and ultrastructure of the cell membrane. Korean J Physiol Pharmacol. 24, 529-543 (2020).

19. Borysova, L. et al. High spatial and temporal resolution $\mathrm{Ca}^{2+}$ imaging of myocardial strips from human, pig and rat. Nature Protocols. Available from: https://doi.org/10.1038/s41596-021-00590-6 (2021).

20. Itzhaki-Alfia, A. et al. Patient characteristics and cell source determine the number of isolated human cardiac progenitor cells. Circulation. 120, 2559-2566 (2009).

21. Kajiura, S. et al. Cryopreservation of the Hair Follicle Maintains Pluripotency of Nestin-Expressing Hair Follicle-Associated Pluripotent Stem Cells. Tissue Eng Part C Methods. 21, 825-831 (2015).

22. Yamane, M. et. al. Hair-Follicle-Associated Pluripotent (HAP) Stem Cells Can Extensively Differentiate to Tyrosine-Hydroxylase-Expressing Dopamine-Secreting Neurons. Cells. 10, 864 (2021). 


\section{Tables}

Table. 1 Primers for qPCR

\begin{tabular}{lll} 
Genes & Forward Primer & Reverse Primer \\
\hline cTnT & CCT GAC GGA GAG AGA GTG GA & CAG AGT CTG CAG CTC GTT CA \\
\hline cTnl & TAT GAC CTG CGT GGC AAG TT & GCC CTC AGG TCC AAG GAT TC \\
\hline MLC-2a & AGG CCA TCC TGA GTG CTT TC & GTC AGG TCC ATG GGT GTC AG \\
\hline GAPDH & GCA TCT TCT TGT GCA GTG CC & GAT GGT GAT GGG TTT CCC GT
\end{tabular}

\section{Figures}
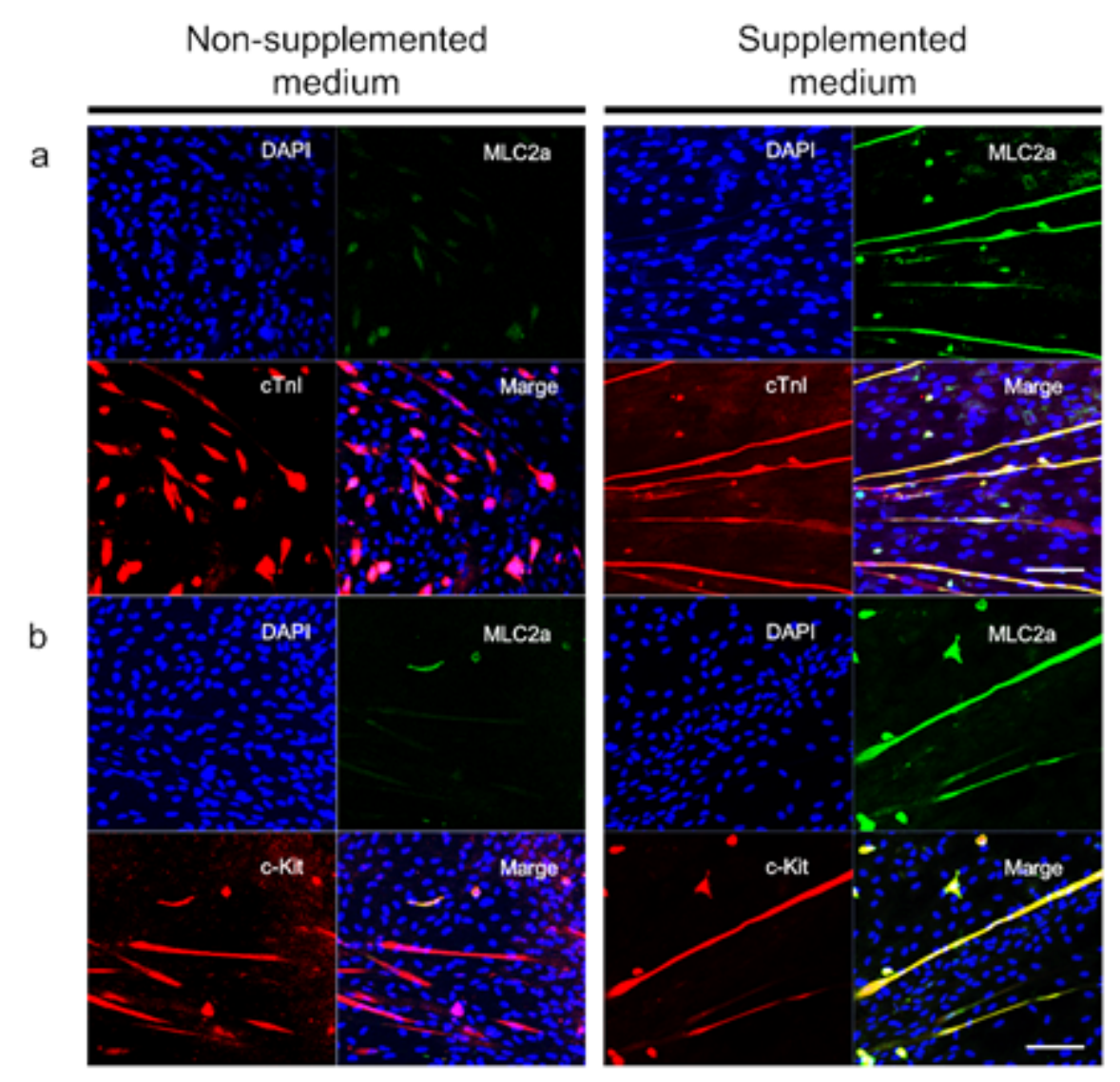

\section{Figure 1}

HAP stem cells simultaneously differentiated to cardiomyocytes and atrial myocytes. The left panels show HAP stem cells cultured in non-supplemented medium. The right panels show HAP stem cells cultured in supplemented medium (pleased see materials and method for ingredients). (a): Immunofluorescence staining shows that HAP stem cells cultured in supplemented medium differentiated to cTnl-positive (red fluorescence) cardiomyocytes and MLC-2a-positive (green fluorescence) atrial myocytes. (b): Immunofluorescence staining shows that HAP stem cells cul-tured in 
supplemented medium differentiated to c-kit-positive cardiomyocytes (red fluorescence) and MLC-2apositive atrial myocytes (green fluorescence). DAPI (blue fluorescence). (Scale bar = $100 \mu \mathrm{m}$ )

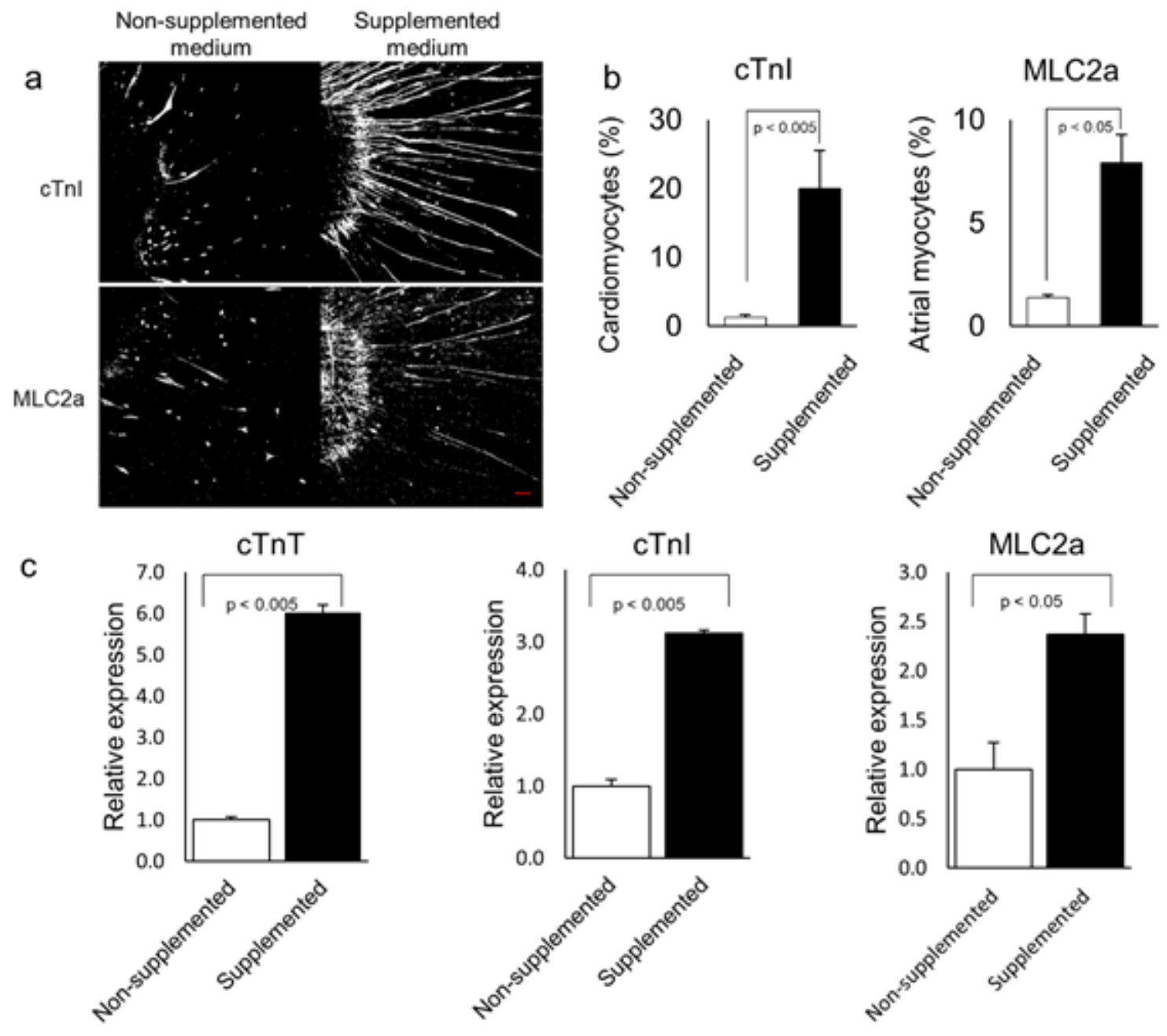

Figure 2

(a): HAP stem cells simultaneously differentiated to cardiomyocytes and atrial myocytes in supplemented medium. (b): The frequency of cTnl-positive cardiomyocytes and MLC-2a-positive atrial myocytes differentiated from HAP stem cells significantly increased in supplemented me-dium compared to non-supplemented medium. (c): qPCR analysis of the effect of supplemented medium on the cardiomyocyte and atrial myocyte-related gene expression. The relative expres-sion of cTnT, cTnl and MLC2a significantly increased in supplemented medium compared to non-supplemented medium. Note: cTnT, cardiac troponin T; cTnl, cardiac troponin I; MLC-2a, myosin light chain. 


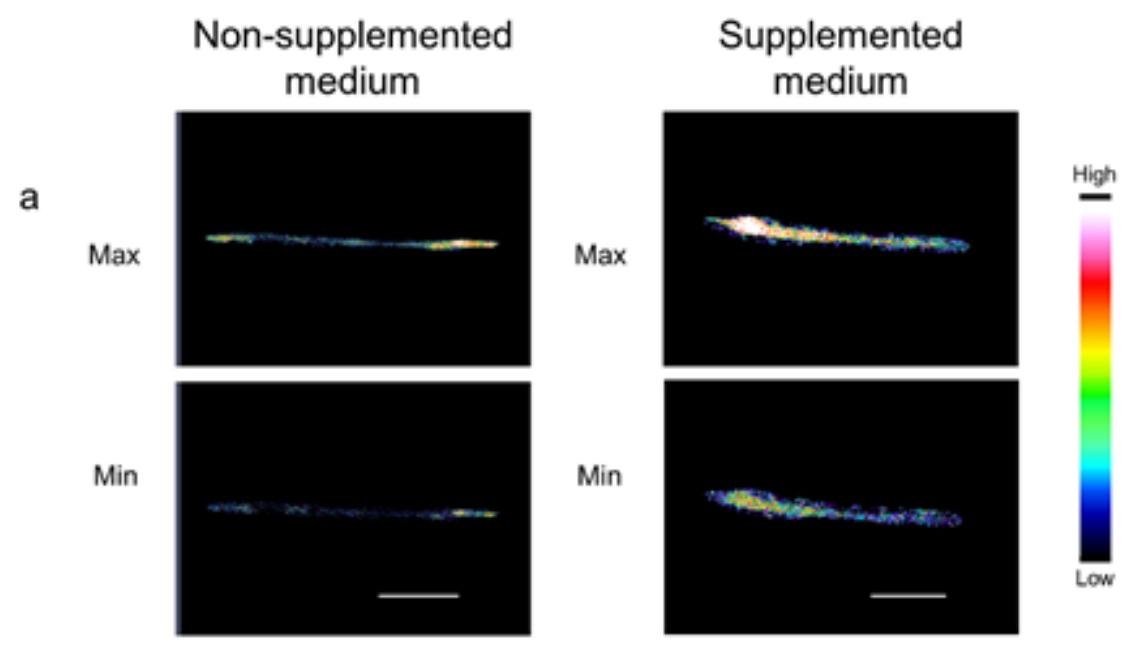

b

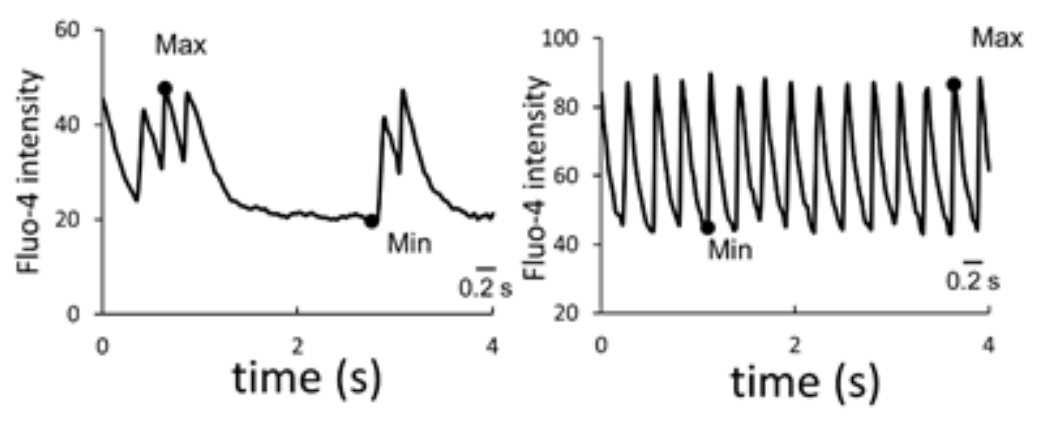

Figure 3

Intercellular $\mathrm{Ca} 2+$ imaging of cardiomyocytes differentiated from rat HAP stem cells. Phase con-trast image of cardiomyocytes loaded with Fluo 4-AM. Images were obtained for 200 frames at an interval of 5.0 msec. (a). Scale bar $=100 \mu \mathrm{m}$.

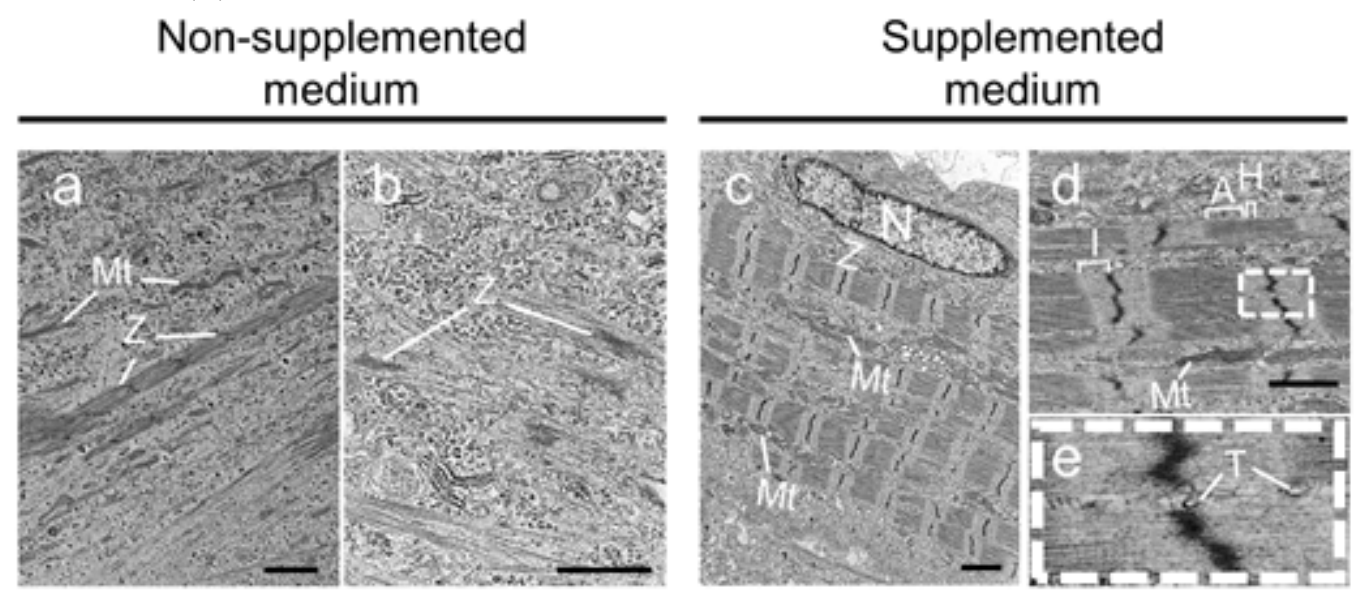

\section{Figure 4}

Electron microscopy images of cardiomyocytes differentiated from HAP stem cells. The left panels show HAP stem cells cultured in non-supplemented medium $(a, b)$. The right panels show HAP stem cells cultured in supplemented medium (c, d, e). Myofibrils with Z-bands (Z), A-bands (A), I-bands (I), H-zone 
$(H)$, mitochondria $(M t)$, T-tubule like structures $(T)$, nucleus $(N)$. e is a magnified image of $d$. Image a, c $($ Scale bar $=2 \mu \mathrm{m})$, Image $b, \mathrm{~d}($ Scale bar $=1 \mu \mathrm{m})$.

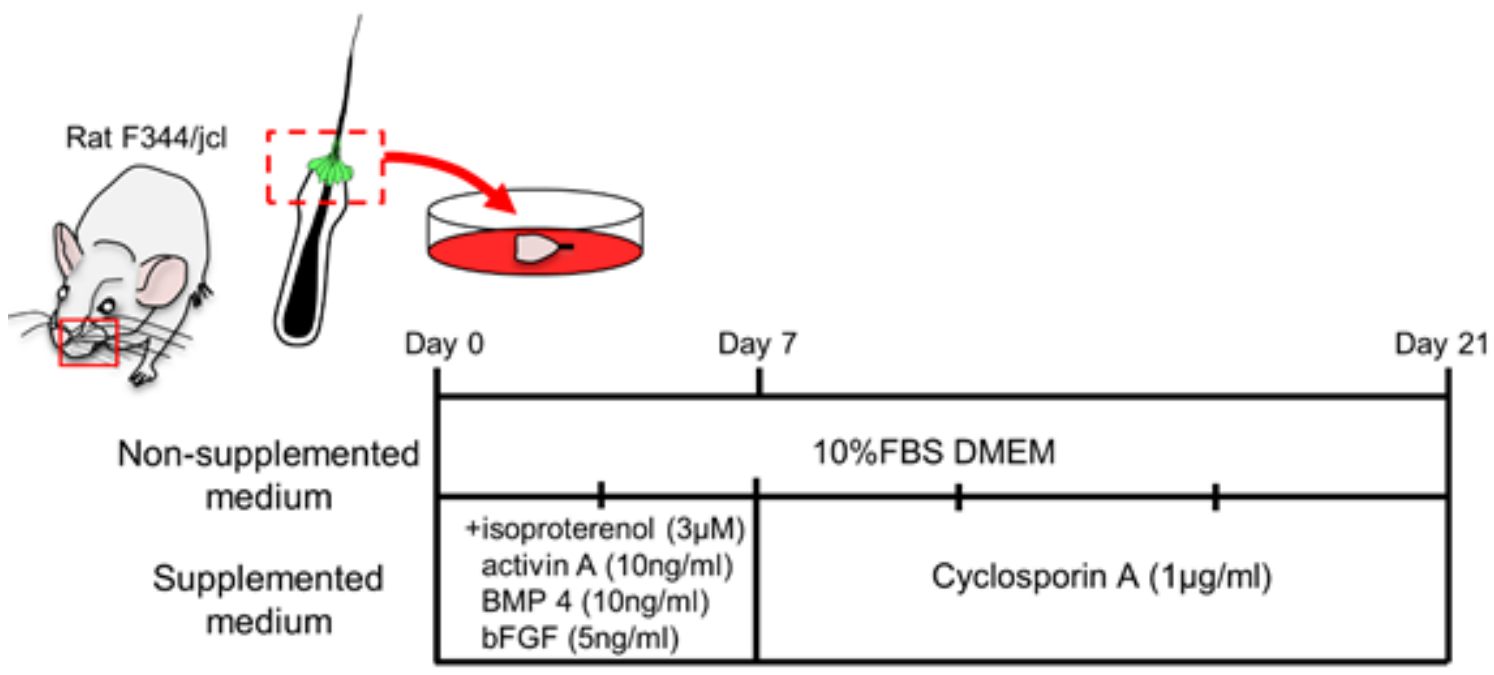

\section{Figure 5}

Scheme for the simultaneous differentiation of cardiomyocytes and atrial myocytes from HAP stem cells. HAP stem cells were initially cultured from the upper part of the rat vibrissa hair follicle (box in cartoon) in $10 \%$ FBS-DMEM supplemented with iso-proterenol, activinA, bone morphogenetic protein 4 (BMP4) and basic fibroblast growth factor (bFGF) for 7 days, which was changed to $10 \%$ FBS-DMEM supple-mented with cyclosporin A (CSA) for 14 days. (Total 21 days)

\section{Supplementary Files}

This is a list of supplementary files associated with this preprint. Click to download.

- S1.movie.mp4

- S2movienonsupplemented.avi

- S2moviesupplemented.avi 